\title{
DIGITAL HALFTONING ALGORITHM USING VISUAL-OPTIMIZED BINARY PATTERNS
}

\author{
H. J. Lee, Y. J. Yoo and H. W. Park \\ Department of Information and Communication Engineering \\ Korea Advanced Institute of Science and Technology \\ 207-43, Cheongryangri, Dongdaemun-Gu, Seoul, Korea \\ E-mail: hwpark@sicom.kaist.ac.kr
}

\begin{abstract}
The digital halftoning is a gray level rendition in the devices that have the limited gray representation capabilities. The major evaluation points of the digital halftoning methods are the image quality and the processing speed. A new halftoning method using non-thresholding process is proposed, which simply constructs a halftoning database to achieve real time halftoning as well as optimal image quality. The proposed algorithm is constructed by two major parts. First one is to generate well-distributed binary patterns for every gray level. A contrast sensitivity function (CSF) is used as a filter to distribute void and cluster regions and as a criterion of iteration. Second one is to index the halftoning database to image pixel value. The halftoning database consists of the well-distributed binary patterns. By the proposed algorithm, halftoned images can be obtained faster than the other algorithms and produces similar image quality to those of the max energy mask [1].
\end{abstract}

\section{INTRODUCTION}

There are so many kinds of algorithms for digital halftoning. Among them, ordered dithering [2] and error diffusion algorithms [3] are widely used in today. The ordered dithering is a simple thresholding process with welldefined ordered matrix. It is good for real time processing, but has some shortcomings such as cross pattern, macrodot and false contouring. The error diffusion is neighborhood processing that requires longer computation time than the ordered dithering. The error means a difference between the original gray value and the thresholded value. It produces good image quality having characteristics of blue noise, but it also produces some correlated artifacts such as silk worm patterns. A recent approach is the model based halftoning [4]. The sensitivity of human eyes is dull for high and extremely low frequency ranges. These characteristics of human visual system admit the digital halftoning to be widely used for printing.
A contrast sensitivity function (CSF) was developed to simulate the optimum encoding of still image by Mannos and Sakrison [5]. The CSF has been improved to incorporate the other characteristics of the eye. Daly introduced an advanced CSF model including the nature of angular dependency of the human visual system and it has been utilized by J. Sullivan et al. [6]. Daly's CSF model is used in this paper because it is not complex and always gives a reasonable result for our research. For an alternative of these algorithms, Mitsa proposed a blue noise mask [7] and a max energy mask which took advantages of both ordered dithering and error diffusion. These masks are constructed from the cumulative array, which is obtained from locally aperiodic and isotropic binary dot profiles for every gray level. Each dot profile is sequentially constructed with a blue noise filter or an exponential-type filter. Despite some correlated artifacts like silk-worm pattern, the image quality is very pleasing. However, the mask construction is a little bit complex and these algorithms still require thresholding process.

The proposed algorithm is the non-thresholding halftoning. At first, this algorithm produces a pseudo random distributed binary pattern for each gray level. The next step is to make the initial randorn patterns well-distributed visually. A fast algorithm using Daly's CSF is proposed to generate the well-distributed binary patterns. CSF is used as a filter to find the void and cluster regions and a criterion of iteration for well distribution. The halftoning database is a set of the well-distributed binary patterns for every gray level. The halftoning process is only to index the halftoning database to image pixel value depending on the location and the gray level of current processing pixel. The halftoned image may include clustered dot artifacts at somewhere. To avoid the artifacts, a swapping technique is introduced.

Section 2 of this paper describes a procedure to generate the well-distributed pattern. The proposed halftoning procedure is given in section 3 . In section 4 , one dimensional swapping technique is introduced to avoid some clustered artifacts. Finally, experimental results and conclusion are also presented in sections 5 and 6 , respectively. 


\section{WELL-DISTRIBUTED BINARY PATTERN}

A binary pattern is generated by psudo random pattern generator and pattern distributor. The psudo random pattern generator makes an initial binary pattern for each gray level. The pattern distributor scatters the initial binary pattern to be well-distributed binary pattern

\subsection{Psudo-random pattern generator}

At first, two random numbers are generated for location value of a black dot. These two numbers are for the horizontal and the vertical coordinates in the binary pattern. Therefore, these two numbers are scaled to the maximum sizes of the binary pattern. If there was already a black dot at that two dimensional location, another random number is repeatedly generated until a given number of black pixels have been placed. The number of black pixels for a gray level $g(i, j)$ of an input image is

$$
N=\frac{N_{i} \times N_{j} \times g(i, j)}{g_{\max }}
$$

where $N$ is the number of black pixels, $N_{i}$ and $N_{j}$ are the coordinates in the horizontal and the vertical directions of the binary pattern, respectively, $g_{\max }$ is the maximum level of $g(i, j)$. The $g_{\max }$ is 255 for 8 bit-per-pixel images. In this paper, both $N_{i}$ and $N_{j}$ have a value of 128 , and 255 for $g_{\max }$

With taking minority and majority pixel concept, the number of iterations for placing black dots can be reduced by half. When less than half of the pixels in the binary pattern are 1's, 1's are the minority pixels and the majority pixels are 0's. The reverse is true when more than half of the pixels are 1's. The first half of psudo random patterns for gray level 0 to $\left(g_{\max }-1\right) / 2$ are generated with above mentioned procedure. However, another half of psudo random patterns for gray level $\left(g_{\max }+1\right) / 2$ to $g_{\text {max }}$ can be generated directly from exchanging 0 's pixels and 1's pixels of the first half patterns.

\subsection{Pattern distributor}

The resulting binary pattern from the psudo random pattern generator may have some clustered and void black pixels at some locations. These undesirable clusters should be scattered for well distribution. Daly's CSF is used in this procedure. A maximum value after the CSF filtering indicates the most clustered location and a minimum value indicates the most void location. By exchanging two dots of the maximum and the minimum values, the binary pattern is more scattered. The binary pattern be- comes well-distributed by iterations of this exchanging process. Here comes a problem with the number of iterations. For automatic stopping of iterations, a cost function is used. The cost function is simply a mean square error between the given gray level and the CSF filtered value.

$$
\cos t=\frac{1}{N_{W} \times N_{H}} \sum_{i=0}^{N_{\mathrm{r}}-1} \sum_{j=0}^{N_{H}-1}(g(i, j)-f(i, j))^{2}
$$

where $N_{H}$ is the width of the binary pattern, $N_{H}$ is the height of the binary pattern, and $f(i, j)$ is the CSF filtered value of the binary pattern. The derivative of the cost function is approximated as follows,

$$
\frac{d \cos t}{d n}=\cos t(n)-\cos t(n-1)
$$

where $n$ is the iteration step number. When the derivative of the cost function is less than a threshold value, which is 1.0 in this paper, the iteration will be stopped.

\section{HALFTONING PROCESS}

The resulting binary pattern obtained in section 2 is tagged with its gray level, with which the halftoning database is constructed. The system architecture for the proposed halftoning process is shown in Fig. 1, and the procedure is given as follows;

(1) For continuous tone image value $g(i, j)$, find the tagged binary pattern with the corresponding gray level, $g$, in the halftoning database.

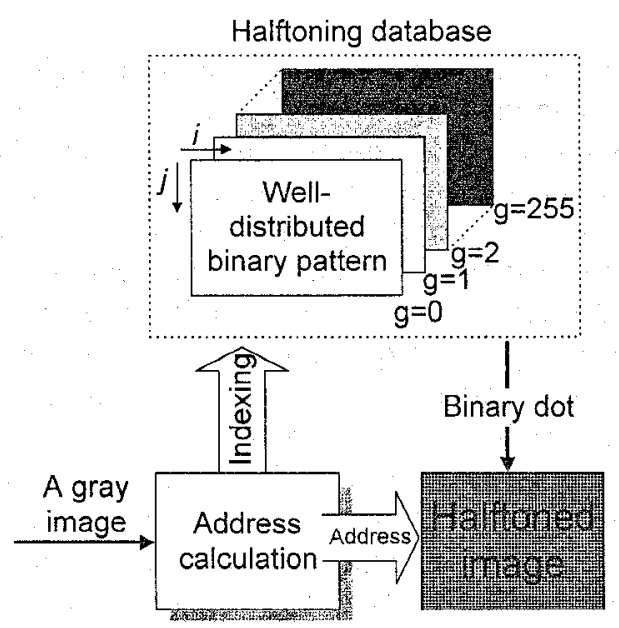

Fig. 1 System architecture for the proposed halftoning process. 
2 Bring a zero or one from the same location $(i, j)$ in the binary pattern. If the size of binary pattern is smaller than that of the original image, the location in the binary pattern is given as ( $i$ modulo $N_{n}, j$ modulo $N_{H}$ ).

3 Apply this zero or one to halftoned image plane at the same location as the location of the original image.

\section{SWAPPING TECHNIQUE}

The halftoned pattern with above processing reveals good results for gray levels under the 100 and over the 140 when $g_{\max }$ is 255 . Because of the independence of each binary pattern, the gray levels near the 128 , half of $g_{\max }$, can be clustered. To avoid this phenomenon, a swapping technique is applied to the halftoned image as a postprocessing. This swapping technique is as follows;

(1) Examine the previous dropped dot in the horizontal direction.

2 If the current dot to be dropped is the same as the previous dot, the current dot in the binary pattern is exchanged with the horizontally next dot in the same binary pattern.

(3) The exchanged dot in the current location is chosen for current pixel of halftoned image. These procedures are repeated for all image pixels.

\section{RESULTS}

Figures 2, 3, 4 and 5 show original images and halftoned images by the proposed algorithm. The original images have the spatial resolution of $512 \times 512$ and the contrast resolution of 8 bits. The perceptual test of the image quality is much better than the ordered dithering and similar to the max energy mask algorithm. The cost value is exponentially decreased as the iteration is progressed. The memory size and the number of computations are important when the algorithm is to be implemented on a hardware platform. The required memory size is $N_{H} \times N_{H} \mathrm{x}$ $\left(g_{\max }+1\right) / 8$ bytes. In this paper, both $N_{\|}$and $N_{I I}$ are 128 and $g_{\max }$ is 255 , so the memory size is about 524 Kbytes. The comparison of the number of computations is given in Table 1. As shown in Table 1, the proposed halftoning algorithm requires only a single bit comparison for swapping.

\begin{tabular}{|l|c|c|c|}
\hline & $\begin{array}{c}\text { Proposed } \\
\text { algorithm }\end{array}$ & $\begin{array}{c}\text { Max energy } \\
\text { mask }\end{array}$ & $\begin{array}{c}\text { Error diffu- } \\
\text { sion(Stucki) }\end{array}$ \\
\hline Multiplication & None & None & 12 \\
\hline Summation & None & None & 12 \\
\hline Subtraction & None & 1 & 1 \\
\hline Comparison & 1 (bit) & 1 & 1 \\
\hline
\end{tabular}

Table 1 Comparison of computation requirements for a pixel.

\section{CONCLUSION}

The proposed digital halftoning is based on the contrast sensitivity function and reduces the amount of computations compared with other halftoning algorithms. For real implementation, this algorithm needs small memory and simple operations. Most of all, the main feature of this algorithm is a combination of the past two independent studies. One is visually optimization of binary pattern, and the other is real time processing. However, this algorithm also has some problems. The one dimensional swapping could not completely compensate the clustered artifacts, especially in the vertical direction. Two dimensional swapping can overcome this artifact but computation complexity should be increased.

\section{REFERENCES}

[1] T. Mitsa and P. Brathwaite, "Wavelets as a tool for the construction of a halftone screen," Proc. SPIE: Human vision, Visual Processing and Digital Display VI, vol. 2411, pp. 228-238, 1995.

[2] B. E. Bayer, "An optimum method for two-level rendition of continuous-tone pictures," Proc. IEEE Int. Conf. Commun. pp. 26.11 - 26.15, 1972.

[3] R. W. Floyd and L. Steinberg, "Adaptive algorithm for spatial grey scale," SID Int. Sum. Digest of Tech. Papers, pp. 36-37, 1975.

[4] T. N. Pappas and D. L. Neuhoff, "Least-square modelbased halftoning," Proc. SPIE: Human Vision, Visual Processing and Digital Display III, vol. 1666, pp. 165176, 1992.

[5] J. L. Mannos and D. J. Sakrison, "The effects of a visual fidelity criterion on the encoding of images," IEEE Trans. Info. Theory, vol. IT-20, no. 4, pp. 525-536, 1974. [6] J. Sullivan, L. Ray and R. Miller, "Design of minimum visual modulation halftone patterns," IEEE Trans. on Systems, Man, and Cybernetics, vol. 21, no.1, pp. 33-8, 1991. [7] T. Mitsa and K. J. Parker, "Digital halftoning using a blue noise mask," Proc. ICASSP 91, pp. 2809-2812, 1991. 


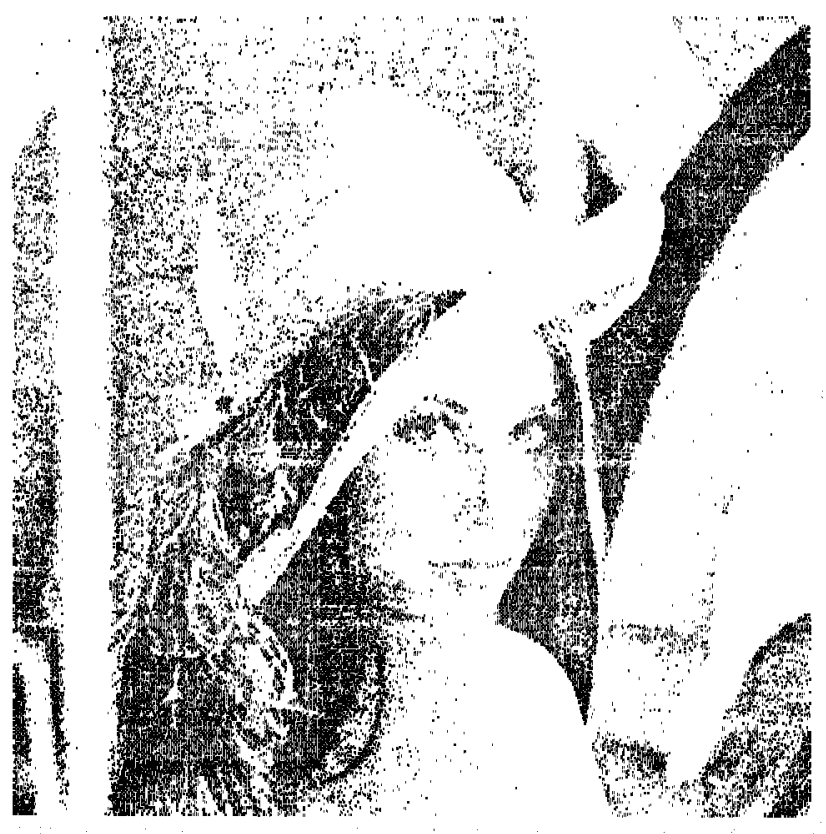

Fig. 2 Original Lena image with $512 \times 512$ pixel and 256 gray levels.

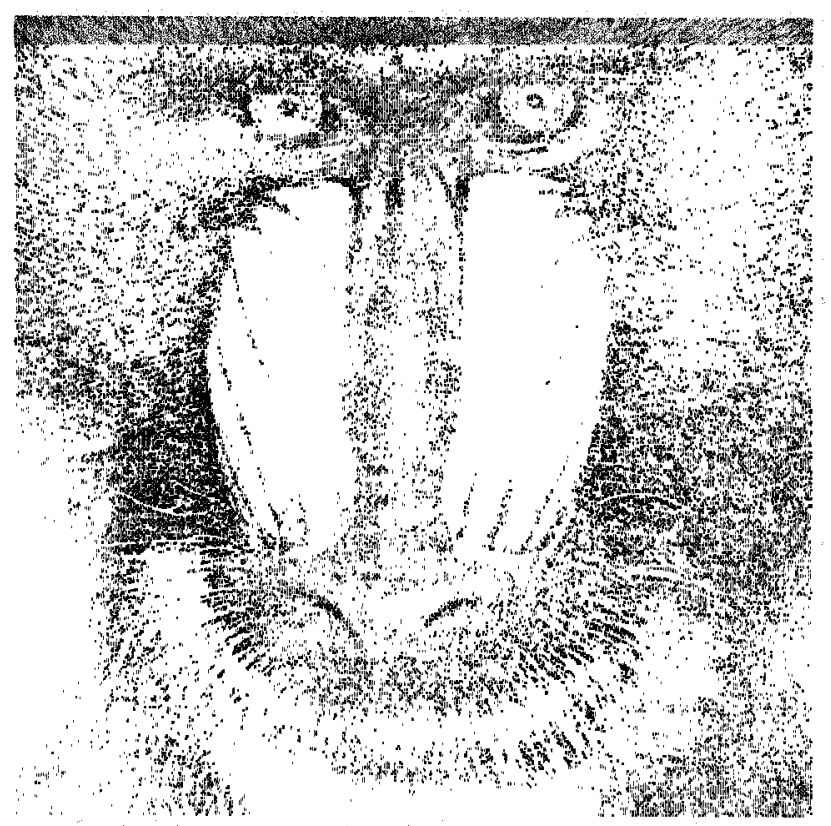

Fig. 4 Original Baboon image with $512 \times 512$ pixel and 256 gray levels.

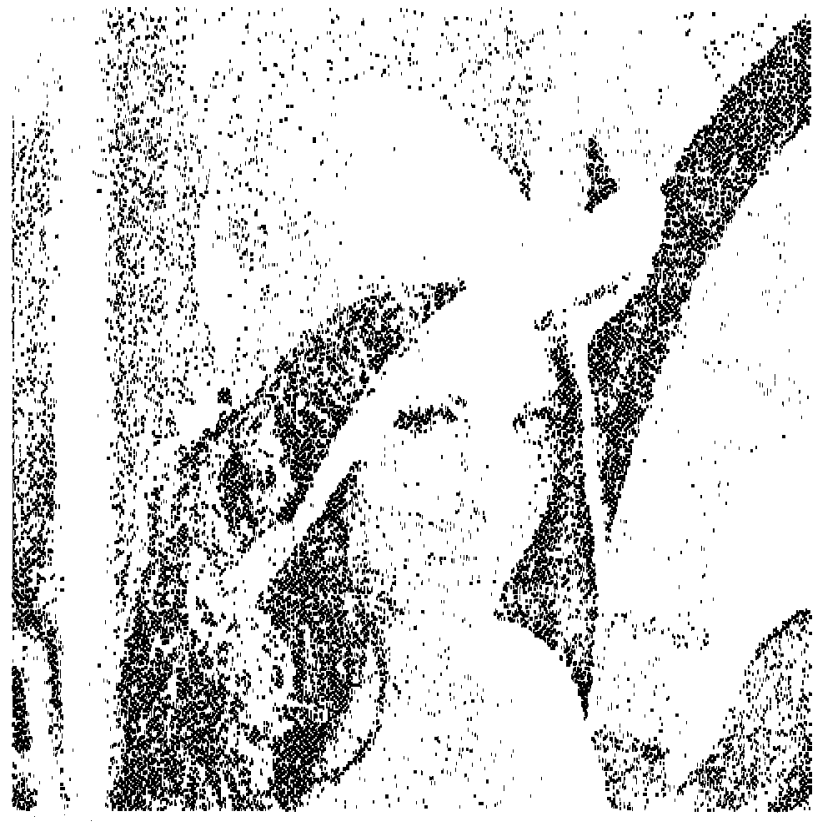

Fig. 3 Halftoned Lena image generated by the proposed algorithm.

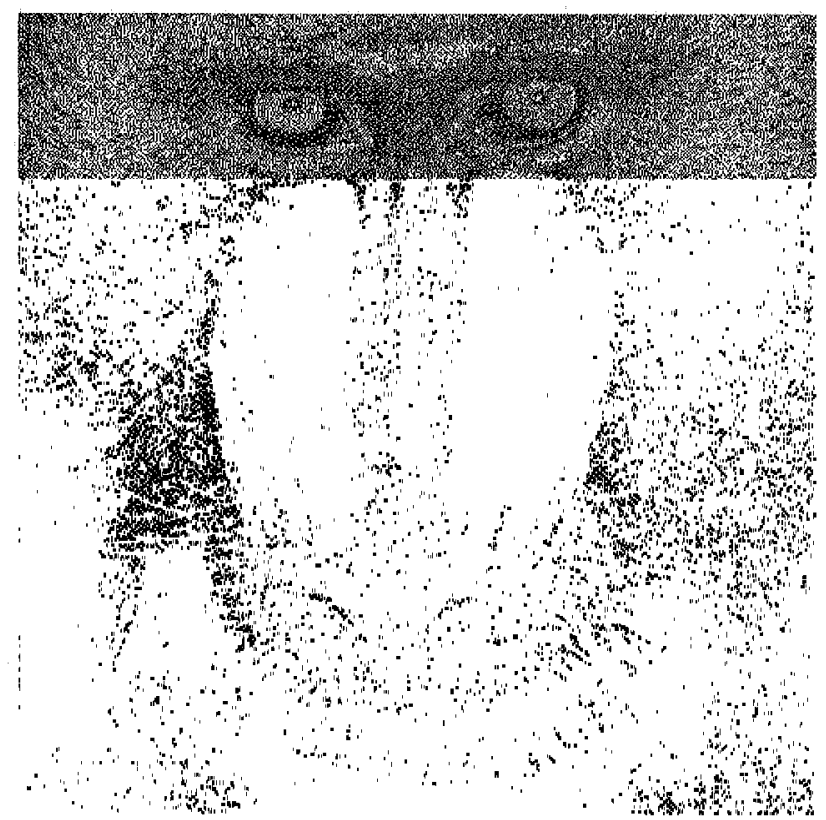

Fig. 5 Halftoned Baboon image generated by the proposed algorithm. 